\title{
Immunotherapy combinations transform the treatment paradigm for advanced renal cell carcinoma
}

\author{
Justin A. Shaya, Rana R. McKay \\ Department of Medicine, University of California San Diego, Moores Cancer Center, La Jolla, CA, USA \\ Correspondence to: Rana R. McKay, MD. Assistant Professor of Medicine, Department of Medicine, University of California San Diego, Moores \\ Cancer Center, 3855 Health Science Road, La Jolla, CA 92093, USA. Email: rmckay@health.ucsd.edu. \\ Provenance: This is an invited article commissioned by the Section Editor Dr. Xiao Li (Department of Urology, Jiangsu Cancer Hospital, Jiangsu \\ Institute of Cancer Research, Nanjing Medical University Affiliated Cancer Hospital, Nanjing, China). \\ Comment on: Motzer RJ, Rini BI, McDermott DF, et al. Nivolumab plus ipilimumab versus sunitinib in first-line treatment for advanced renal cell \\ carcinoma: extended follow-up of efficacy and safety results from a randomised, controlled, phase 3 trial. Lancet Oncol 2019;20:1370-85.
}

Submitted Dec 17, 2019. Accepted for publication Dec 19, 2019.

doi: $10.21037 /$ atm.2019.12.130

View this article at: http://dx.doi.org/10.21037/atm.2019.12.130

Over the past 15 years, the treatment options for patients with advanced renal cell carcinoma (RCC) have undergone tremendous transformation. From single agent vascular endothelial growth factor (VEGF) targeted therapy, to now immunotherapy combinations, survival is improving given the results of landmark clinical trials which have expanded treatments for patients. CheckMate-214 is a practice changing phase III trial that has heralded the use of combination immunotherapy in frontline management for clear cell RCC. Results of this trial were first published in the New England Fournal of Medicine in 2018 (1) and an extended follow-up was published in Lancet Oncology in October 2019 (2). In this commentary, we will review the trial design, endpoints, patient population, and quality of life (QOL) and toxicity data. Additionally, we will place the results of this study in the context of other immunotherapy combination trials. Lastly, we will highlight upcoming studies and pending questions that will inform the design of future clinical trials.

\section{Trial design and endpoints}

CheckMate-214 was an open-label, phase III trial of the combination of nivolumab plus ipilimumab versus sunitinib in patients with advanced RCC. Nivolumab is an antiPD-1 monoclonal antibody, ipilimumab is an anti-CTLA-4 monoclonal antibody, and sunitinib is a VEGF receptor tyrosine kinase inhibitor. The co-primary endpoint was overall survival (OS), progression-free survival (PFS), and objective response rate (ORR) as assessed by independent radiology review in International Metastatic RCC Database Consortium (IMDC) intermediate and poor-risk patients.

At a median follow-up of 25.3 months, the combination of nivolumab and ipilimumab resulted in a statistically significant improvement in OS [18-month OS of $75 \%$ vs. 60\%, hazard ratio (HR): 0.64] (1). With an extended median follow-up of 32.4 months in the updated analysis (2), this OS benefit remained statistically significant (median OS not reached vs. 26.6 months, HR: 0.66). It is worthwhile to highlight the impact of immunotherapy combinations on PFS, which may not serve as a suitable surrogate endpoint for OS for ipilimumab/nivolumab. In the original study analysis, while median PFS, as assessed by independent review, was numerically higher in the ipilimumab/nivolumab arm compared to the sunitinib arm, this difference did not reach statistical significance. In the updated analysis, investigator assessment of PFS, which more likely reflects real-world practice, was presented. While the median PFS for both arms were nearly identical, at 9 months from randomization, there is a clear separation of the curves and superior PFS with ipilimumab/nivolumab which was statistically significant (HR: 0.77). This suggests the durability of benefit to ipilimumab/nivolumab.

Objective responses on this study were assessed by Response Evaluation Criteria in Solid Tumors version 1.1 which has its pitfalls given that immune checkpoint 
inhibitors have unique patterns of response which are not fully captured by traditional response criteria (3). Nonetheless, the ORR as assessed by independent review and investigator assessment in the intention-totreat population were similar and improved compared to sunitinib (39\% vs. $32 \%$ for ipilimumab/nivolumab vs. sunitinib by independent radiology review; $41 \%$ vs. $34 \%$ for ipilimumab/nivolumab $v s$. sunitinib by investigator assessment).

While OS remains a gold standard, additional surrogate endpoints in the context of immunotherapy are worthwhile to mention including complete response (CR) rate, durability of response, and the more recent novel endpoint termed treatment-free survival (TFS). TFS, with or without toxicity, describes the time from cessation of therapy to time of subsequent therapy or death $(4,5)$. In the updated analysis, the CR rate with ipilimumab/nivolumab was $11 \%$ with $88 \%$ of patients maintaining a CR at last follow-up. The median time-to-response was early at 2.8 months and the median time to confirmed CR was 7.6 months. In a subsequent analysis of TFS presented at the Kidney Cancer Association 2019 meeting (4), at 36-month, among intermediate and high-risk patients, $16 \%$ of patients receiving ipilimumab/nivolumab were off treatment compared to $8 \%$ of patients on sunitinib. The mean TFS free from grade 3 or greater treatment-related adverse events was $5.5 v s .2 .8$ months with ipilimumab/nivolumab and sunitinib, respectively.

\section{Patients enrolled and subset analyses in distinct patient populations}

The study was largely conducted in the United States, Canada, and Europe. Patients enrolled in the trial had previously untreated RCC with a clear cell component and all IMDC risk groups were permitted. In the intentionto-treat population, approximately $20 \%$ of patients were favorable-risk, $60 \%$ were intermediate-risk, and $20 \%$ were poor-risk. With the evolving role of cytoreductive nephrectomy, $81 \%$ of patients had undergone a prior nephrectomy. PD-L1 status was available on archival tissue from $90 \%$ of patients and $24 \%$ had tumors which were PDL1 positive.

A post-hoc exploratory analysis was conducted in patients with favorable-risk disease. While OS was not statistically different between the arms in favorablerisk patients, the HR for death favored sunitinib in the original analysis (HR: 1.45) and with extended follow- up, the HR was 1.22 and remained non-significant. In the updated analysis, there was no significant difference in ORRs between the treatment arms in the favorablerisk patients $(39 \%$ vs. $50 \%, \mathrm{P}=0.14)$ and CRs were numerically higher with ipilimumab/nivolumab ( $8 \%$ vs. $4 \%)$. These data suggest that favorable-risk patients may derive similar benefit from ipilimumab/nivolumab and sunitinib. As patients with favorable-risk disease have prolonged survival, evaluating TFS without toxicity is clinically relevant and was 9.4 compared to 2.6 months with ipilimumab/nivolumab compared to sunitinib (4).

An exploratory post-hoc analysis was also conducted in patients with sarcomatoid differentiation (6). The presence of sarcomatoid differentiation is associated with aggressive disease and poor prognosis (7). Overall, 112 patients had a component of sarcomatoid differentiation and with ipilimumab/nivolumab, the ORR and CRs were more pronounced $(57 \%$ vs. $19 \%, \mathrm{P}<0.0001 ; 18.3 \%$ vs. $0 \%)$ and OS was longer in these patients (median OS of $31.2 v s$. 13.6 months, HR: 0.55).

\section{QOL and adverse events}

The side effect profile of checkpoint inhibitors differs from that observed with VEGF targeted therapy given the distinct mechanisms of action of these agents. Grade 3-4 treatment-related toxicities were less frequent with ipilimumab/nivolumab compared to sunitinib (47\% vs. $64 \%)$. Additionally, while sunitinib is associated with more chronic toxicity that can impact QOL and tolerance, most grade 3-4 treatment-related adverse events associated with ipilimumab/nivolumab occurred early and resolved within 6 months of treatment onset, with the exception of endocrine related toxicities requiring hormonal supplementation. Nearly one in every $3-4$ patients (29\%) will require high-dose steroids for adverse event management and a high index of suspicion is warranted with clear instruction about toxicity to patients and the clinical care team. As guidelines are developed and refined to instruct clinicians on the appropriate management of immune-mediated adverse events, communication with patients about expectations of treatment and development of clinical workflows will be important to expeditiously diagnosis and treat immune-mediated adverse events.

In a subsequent analysis of health-related QOL (HRQOL) data from CheckMate-214 (8), patient-reported outcomes were significantly better with ipilimumab/ nivolumab as demonstrated among multiple QOL 
Table 1 Frontline immunotherapy trials

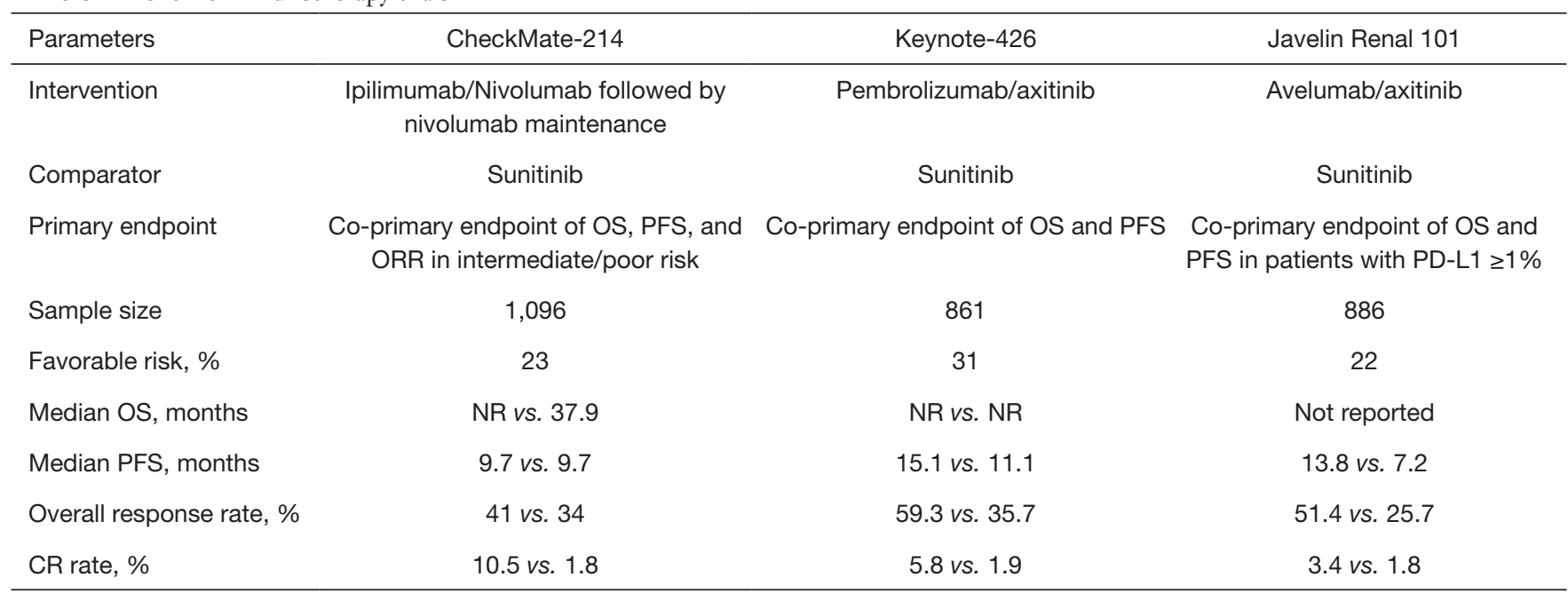

OS, overall survival; PFS, progression-free survival; ORR, objective response rate; NR, not reached; CR, complete response.

instruments including the Functional Assessment of Cancer Therapy- General score (HR: 0.63) and the EuroQol5D-3L score (HR: 0.75). Taken together with the efficacy outcomes, these data highlight that patients are living longer and better with the combination of ipilimumab/ nivolumab.

\section{Additional frontline immunotherapy combinations}

While ipilimumab/nivolumab was the first immunotherapy combination to enter the frontline space for patients with advanced RCC, two additional landmark studies, Keynote-426 and Javelin Renal 101, have informed frontline treatment options (Table 1). Given that the VEGF pathway may contribute to immune evasion (9) and the single agent activity of both VEGF targeted agents and checkpoint blockade, there is rationale for the combination of PD-1 blockade and VEGF inhibition.

Keynote-426 was an open-label, phase III trial of pembrolizumab, an anti-PD-1 monoclonal antibody, plus axitinib compared to sunitinib in previously untreated, advanced clear cell RCC (10). Unlike CheckMate-214, the trial co-primary endpoint was OS and PFS in the overall population. At a median follow-up of 12.8 months, the combination of pembrolizumab/axitinib resulted in improved ORR (59.3\% vs. $35.7 \%)$, PFS (15.1 vs. 11.1 months), and OS (12-month OS of $89.9 \%$ vs. $78.3 \%$ ) compared to sunitinib with a significant HR for death of 0.53. Subgroup analysis across IMDC risk groups favored pembrolizumab/axitinib, including those with favorablerisk disease. Grade 3 or greater treatment-related adverse events were present in the majority of the patients in both study arms (63\% vs. $58 \%$ with pembrolizumab/axitinib vs. sunitinib) with higher rates of transaminase elevations and diarrhea with pembrolizumab/axitinib. The rate of steroid use was not reported and QOL data are not yet available. Of note, a larger proportion of patients in the trial were treated outside of the United States and Europe, impacting access to post-progression therapies, and the trial included a larger proportion of patients with favorable-risk disease $(31 \%)$, as reflected in the prolonged PFS in the control arm. While cross trial comparisons are limited given the differing patient populations in these studies, the ORR with pembrolizumab/axitinib was higher than that observed with ipilimumab/nivolumab, however CRs were higher with ipilimumab/nivolumab.

Javelin Renal 101 was an open-label, phase III trial of avelumab, an anti- PD-L1 monoclonal antibody, plus axitinib compared to sunitinib in previously untreated advanced clear cell RCC (11). Unlike the prior trials, the trial co-primary endpoint was OS and PFS in PD-L1 positive tumors, defined as a PD-L1 expression of $1 \%$ or greater within the tumor. $22 \%$ of patients had favorable-risk disease and $63 \%$ were PD-L1 positive. At a median followup time of 11.6 months, there was a statistically significant improvement in PFS (13.8 vs. 7.2 months) and ORR (55.2\% vs. $25.5 \%$ ) with avelumab/axitinib compared to sunitinib. The CR rate was the lowest with this combination at 3.4\% in the overall population. With only 81 events, OS data 
are still immature with this combination. Compared to Keynote-426, more patients were enrolled in United States, Canada, and Western Europe compared to other geographic areas and this may play a role in access to post-progression therapies and the impact of subsequent treatments on OS. Subset analyses for PFS benefit across all IMDC risk groups favored avelumab/axitinib. Grade 3 or greater treatmentrelated adverse were similar between the arms. Though typically low grade and reversible, infusion-related reactions were more common with avelumab.

\section{Selection of frontline treatment}

The approval in the United States of now three frontline immunotherapy options has created a clinical dilemma regarding the optimal regimen for patients given the lack of level I comparative data of these three options. While the IMDC criteria were initially developed in the targeted therapy era to inform prognosis, they have been applied to trials of immunotherapy despite validation in this context. Nonetheless, these criteria are clinically relevant and allow for risk stratification of patients. These and other clinical factors will certainly play a role in therapy selection. Underlying comorbidities such as autoimmune disease or cardiovascular disease are important to consider when deciding on immunotherapy and VEGF targeted therapy combinations. Practical considerations include mode of drug administration and frequency of infusions. Additionally, toxicities and tolerability of dual immunotherapy $v s$. immunotherapy/VEGF inhibitor are important to consider as CheckMate-214 is the only trial to report improvements in patient reported QOL compared to sunitinib.

Questions remain regarding the role of frontline single agent VEGF inhibitor or checkpoint inhibitor. Cabosun $(12,13)$ was a phase II trial of frontline cabozantinib $v s$. sunitinib in intermediate and poor-risk patients. The primary endpoint was PFS and compared to sunitinib, there was a statistically significant improvement in PFS of $8.2 \mathrm{vs}$. 5.6 months. There was no significant OS benefit although the HR for survival was 0.8 and the authors noted that the study was not sufficiently powered to detect OS differences. Keynote-427 $(14,15)$ was a single arm, phase II trial of frontline pembrolizumab in advanced clear cell RCC (cohort A) and non-clear cell RCC (cohort B). The results of cohort A were most recently presented at the European Society for Medical Oncology (ESMO) meeting in 2019 with an ORR of $36 \%$ and PFS of $37.6 \%$ at 12 months (15). While immunotherapy combination regimens are the preferred regimens based on efficacy, patient factors may lead one to consider single agent VEGF inhibition or immunotherapy such as a contraindication to therapy, performance status, or concerns for tolerability of treatment.

Biomarkers that inform tumor biology will be critical to improve therapy selection for patients. Although PD-L1 expression has prognostic significance, its role as a predictive biomarker in RCC is lacking. Additional biomarkers are warranted to improve therapy selection. The IMmotion 150 trial, a phase II, multi-center trial of atezolizumab with and without bevacizumab $v$ s. sunitinib in advanced clear cell RCC, examined the role of predictive biomarkers in understanding response to immunotherapy and VEGF inhibition (16). The trial developed angiogenesis, T-effector/IFN- $\gamma$ response, and myeloid inflammatory gene expression signatures and correlated the signature with outcomes. Patients with an angiogenesis ${ }^{\text {high }}$ signature had improved responses to sunitinib over atezolizumab/ bevacizumab and atezolizumab alone. The opposite was seen in patients with anangiogenesis ${ }^{\text {low }}$ signature with greater responses to atezolizumab and atezolizumab/ bevacizumab. This study suggests that there are likely molecularly defined subtypes of RCC that have differential responses to anti-VEGF therapy and immunotherapy. Identifying and standardizing the biomarkers to predict these subtypes will aid in selection of the ideal frontline regimen.

Several additional trials are currently ongoing and likely to influence and complicate the treatment landscape for RCC. The TITAN-RCC trial (NCT02917772) is a novel phase II, adaptive immunotherapy trial. Patients in this trial were treated with nivolumab induction for 8 cycles and depending on response, either continued on nivolumab maintenance or received an ipilimumab boost if they had stable disease (SD) or progressive disease (PD). Initial data were presented at ESMO 2019 demonstrating that ipilimumab added an approximately $10 \%$ improvement in ORR (17). Another adaptive phase II trial, OMNIVORE (NCT03203473), is examining a response-based approach in which patients are treated with nivolumab for 4-6 months and therapy is adapted depending on response. Patients with a complete or partial response (PR) will have treatment discontinued while those with SD or PD will receive the addition of ipilimumab (18). There are several ongoing trials examining other frontline immunotherapy/VEGF inhibitor combinations. These include Checkmate-9ER (NCT03141177), CLEAR (NCT02811861), COSMIC-313 (NCT03937219), and PDIGREE (NCT03793166) (Table 2). 
Table 2 Ongoing trials in frontline therapy

\begin{tabular}{|c|c|c|c|c|}
\hline Trial & $\begin{array}{l}\text { Trial registry } \\
\text { number }\end{array}$ & Intervention & Comparator & Primary Endpoint \\
\hline TITAN-RCC & NCT02917772 & $\begin{array}{l}\text { Nivolumab (adaptive); nivolumab/ } \\
\text { ipilimumab if progression }\end{array}$ & NA & ORR \\
\hline OMNIVORE & NCT03203473 & $\begin{array}{l}\text { Nivolumab (adaptive); nivolumab/ } \\
\text { ipilimumab if progression }\end{array}$ & NA & $\begin{array}{l}\text { Number of subjects with persistent PR/ } \\
\text { CR after nivolumab discontinuation } \\
\text { (arm A) }\end{array}$ \\
\hline CheckMate-9ER & NCT03141177 & Cabozantinib/nivolumab & Sunitinib & PFS \\
\hline COSMIC-313 & NCT03937219 & $\begin{array}{l}\text { Cabozantinib/nivolumab/ipilimumab } \\
\text { followed by cabozantinib/nivolumab } \\
\text { maintenance }\end{array}$ & $\begin{array}{l}\text { Ipilimumab/nivolumab, } \\
\text { followed by nivolumab } \\
\text { maintenance }\end{array}$ & PFS \\
\hline \multirow[t]{2}{*}{ CLEAR } & NCT02811861 & Lenvatinib/everolimus & Sunitinib & PFS \\
\hline & & Lenvatinib/pembrolizumab & & \\
\hline
\end{tabular}

NA, not applicable; ORR, objective response rate; CR, complete response; PR, partial response; SD, stable disease; PD, progressive disease; OS, overall survival; PFS, progression-free survival.

At this time, the frontline space in advanced RCC is ripe with new options and the potential for even more in the near future. Questions remain about the optimal sequencing of therapies, and the role of immunotherapy following progression on an immunotherapy combination. As patients are living longer, the bar has been elevated to demonstrate superiority over the changing standard of care. Nevertheless, the results of the updated analysis of CheckMate-214 show that combination immunotherapy holds the promise of survival benefit, durable response, and improved QOL on therapy in advanced RCC.

\section{Acknowledgments}

We thank the patients and their families and caregivers for participating in the clinical trial.

\section{Footnote}

Conflicts of Interest: RR McKay reports a consultant/advisory role with Bristol Myers Squibb/Pfizer, Exelixis, Janssen, Novartis, and Tempus and institutional research funding from Bayer and Pfizer. JA Shaya has no conflicts of interest to declare.

Ethical Statement: The authors are accountable for all aspects of the work in ensuring that questions related to the accuracy or integrity of any part of the work are appropriately investigated and resolved.

\section{References}

1. Motzer RJ, Tannir NM, McDermott DF, et al. Nivolumab plus ipilimumab versus sunitinib in advanced renal-cell carcinoma. N Engl J Med 2018;378:1277-90.

2. Motzer RJ, Rini BI, McDermott DF, et al. Nivolumab plus ipilimumab versus sunitinib in first-line treatment for advanced renal cell carcinoma: extended follow-up of efficacy and safety results from a randomised, controlled, phase 3 trial. Lancet Oncol 2019;20:1370-85.

3. Beer L, Hochmair M, Prosch H. Pitfalls in the radiological response assessment of immunotherapy. Memo 2018;11:138-43.

4. Regan MM, Atkins MB, Powles T, et al. Treatment-free survival, with and without toxicity, as a novel outcome applied to immuno-oncology agents in advanced renal cell 
carcinoma. Ann Oncol 2019;30:mdz249.067.

5. Regan MM, Werner L, Rao S, et al. Treatment-free survival: a novel outcome measure of the effects of immune checkpoint inhibition-a pooled analysis of patients with advanced melanoma. J Clin Oncol 2019;37:3350-8.

6. McDermott DF, Choueiri TK, Motzer RJ, et al. CheckMate 214 post-hoc analyses of nivolumab plus ipilimumab or sunitinib in IMDC intermediate/poorrisk patients with previously untreated advanced renal cell carcinoma with sarcomatoid features. J Clin Oncol 2019;37:4513.

7. Shuch B, Bratslavsky G, Linehan WM, Srinivasan R. Sarcomatoid renal cell carcinoma: a comprehensive review of the biology and current treatment strategies. Oncologist 2012;17:46-54.

8. Cella D, Grünwald V, Escudier B, et al. Patient-reported outcomes of patients with advanced renal cell carcinoma treated with nivolumab plus ipilimumab versus sunitinib (CheckMate 214): a randomised, phase 3 trial. Lancet Oncol 2019;20:297-310.

9. Ott PA, Hodi FS, Buchbinder EI. Inhibition of immune checkpoints and vascular endothelial growth factor as combination therapy for metastatic melanoma: an overview of rationale, preclinical evidence, and initial clinical data. Front Oncol 2015;5:202.

10. Rini BI, Plimack ER, Stus V, et al. Pembrolizumab plus axitinib versus sunitinib for advanced renal-cell carcinoma. N Engl J Med 2019;380:1116-27.

11. Motzer RJ, Penkov K, Haanen J, et al. Avelumab plus axitinib versus sunitinib for advanced renal-cell carcinoma. N Engl J Med 2019;380:1103-15.

12. Choueiri TK, Halabi S, Sanford BL, et al. Cabozantinib

Cite this article as: Shaya JA, McKay RR. Immunotherapy combinations transform the treatment paradigm for advanced renal cell carcinoma. Ann Transl Med 2019;7(Suppl 8):S385. doi: 10.21037/atm.2019.12.130 versus sunitinib as initial targeted therapy for patients with metastatic renal cell carcinoma of poor or intermediate risk: the alliance A031203 CABOSUN trial. J Clin Oncol 2017;35:591-7.

13. Choueiri TK, Hessel C, Halabi S, et al. Cabozantinib versus sunitinib as initial therapy for metastatic renal cell carcinoma of intermediate or poor risk (Alliance A031203 CABOSUN randomised trial): Progression-free survival by independent review and overall survival update. Eur J Cancer 2018;94:115-25.

14. Donskov F, McDermott DF, Lee JL, et al. KEYNOTE-427 cohort A: pembrolizumab monotherapy as first-line therapy in advanced clear cell renal cell carcinoma (ccRCC). Ann Oncol 2018;29:mdy283.080.

15. Larkin JMG, Tykodi SS, Donskov F, et al. First-line pembrolizumab (pembro) monotherapy in advanced clear cell renal cell carcinoma (ccRCC): updated follow-up for KEYNOTE-427 cohort A. Ann Oncol 2019;30:mdz249.045.

16. Rini BI, Powles T, Atkins MB, et al. Atezolizumab plus bevacizumab versus sunitinib in patients with previously untreated metastatic renal cell carcinoma (IMmotion151): a multicentre, open-label, phase 3, randomised controlled trial. Lancet 2019;393:2404-15.

17. Grimm MO, Schmidinger M, Duran Martinez I, et al. Tailored immunotherapy approach with nivolumab in advanced renal cell carcinoma (TITAN-RCC). Ann Oncol 2019;30:mdz394.051.

18. Wei XX, McKay RR, Gray KP, et al. Optimized management of nivolumab (NIVO) and ipilimumab (IPI) in advanced renal cell carcinoma (OMNIVORE): a response-based phase II study. J Clin Oncol 2018;36:4600. 